\title{
Extracellular vesicles as carriers of viruses
}

\author{
Zach Troyer ${ }^{1}$, John C. Tilton ${ }^{2}$ \\ ${ }^{1}$ Department of Molecular and Comparative Pathobiology, Johns Hopkins University, Baltimore, MD, USA; ${ }^{2}$ Department of Nutrition, Case \\ Western Reserve University, Cleveland, OH, USA \\ Correspondence to: John C. Tilton. Department of Nutrition, Case Western Reserve University, Cleveland, OH, USA. Email: john.c.tilton@case.edu. \\ Comment on: Kerviel A, Zhang M, Altan-Bonnet N. A New Infectious Unit: Extracellular Vesicles Carrying Virus Populations. Annu Rev Cell Dev \\ Biol 202 1;37:171-97.
}

Received: 04 November 2021; Accepted: 16 November 2021; Published: 30 December 2021.

doi: 10.21037/exrna-21-24

View this article at: https://dx.doi.org/10.21037/exrna-21-24

An inescapable conclusion of the past decade of research into extracellular vesicles (EVs) during viral infection is the growing recognition that there is not a categorical division between EVs and enveloped viruses, but rather an extensive and intricate overlap leading to the production of a heterogenous mix of submicron particles. Like EVs, enveloped viruses are small, lipid bilayer-enclosed particles and numerous recent reports have demonstrated significant similarity in physical and biochemical composition between EVs and viral particles (1-3). EVs and many viruses use the same cellular machinery to exit the cell and both are capable of transferring proteins and nucleic acids to target cells, which can have functional downstream effects. Furthermore, in addition to these similarities in size, composition, and biogenesis, there is a growing body of evidence that EVs play a major role during the course of viral infections. EVs released from virally-infected cells carry viral components to nearby uninfected cells, sensitizing them for viral spread $(4,5)$. Alternatively, EVs can be released carrying antiviral proteins allowing nearby cells to prepare their defenses for a potential viral encounter $(6,7)$. In "A New Infectious Unit: Extracellular Vesicles Carrying Virus Populations", Kerviel et al. review a recently recognized phenomenon in which EVs facilitate the spread of viruses by encapsulating multiple virions or naked infectious viral genomes (8), expanding our understanding of the complex overlap between viruses and EVs that plays out at the nanoscale stage.

In "vesicle-mediated en bloc viral transmission", EVs containing non-enveloped viruses, enveloped viruses, or even naked viral genomes are the main carrier of infection and viral transmission rather than individual "free" viral particles. En bloc transmission has been observed in a wide array of viral families, including polioviruses, enteroviruses, Coxsackieviruses, hepatitis A (HAV) and C viruses (HCV), rotaviruses, and polyomaviruses, suggesting a potential evolutionary advantage for the viruses for transmitting via EVs. A defining feature of en bloc transmission is the presence of multiple viruses per EV. This simple observation has several profound implications for viral transmission and the role of EVs. First, evidence suggests that viral infection of cells can be quite inefficient, with many cells failing to become productively infected (9). If a cell is infected by a single virus, the kinetics of viral replication may be slow enough to enable immune responses to develop. Viruses that replicate slowly may be cleared by the immune response before they spread to new cells. In contrast, by packaging multiple viruses per EV, en bloc transmission delivers higher effective MOIs to cells that are infected and increasing the chances of a productive infection and kinetics of replication. Second, the multiple viruses have the potential to recombine and significantly expand viral genetic diversity, which in turn can lead to drug resistance and immune escape. Third, packaging multiple viruses per EV also provides opportunities for complementation of defective proteins or particles, allowing viral mutants to replicate that might otherwise have been initially selected against. This could provide further opportunities for viral mutagenesis by complementing deleterious mutations until additional compensatory mutations that restore fitness are generated.

An additional advantage of en bloc viral transfer delineated by Kerviel et al. is a type of quality control mechanism. Viruses are highly mutagenic due the high 
error rate of most viral polymerases compared to cellular polymerases, coupled with selection pressures. These mutations, along with other inefficiencies of virus assembly and budding, result in most virus families producing large numbers of defective viral particles that are incapable of replication. However, it was found that viruses transmitted en bloc within EVs had less sequence diversity and fewer deleterious mutations (10). At the same time, it was recognized that proper viral assembly at internal replication organelles (typically adjacent to ER or Golgi) was important for packaging of viruses into EV structures (9). If packaging of virions into EVs requires proper viral assembly to occur near the EV packaging site, only virions with correct folding and trafficking of the structural proteins and assembly of the nascent particles will be incorporated into EVs, eliminating a large swath of defective viruses. Moreover, there may be an additional active packaging process in which EV machinery must recognize properly formed viral capsid topologies, thus ruling out virions with assembly defects. These two "quality control checks" prevent highly mutated viruses from being transferred en bloc within EVs, and increase the overall viral fitness of the $\mathrm{EV}$-transmitted viruses compared to free virus particles.

A final major advantage to en bloc transmission is evasion of host immune responses. The ability of the host immune response to rapidly identify and target viruses is the key to preventing systemic viral spread. As detailed by Kerviel and colleagues in their review, the release of viruses in EVs is a non-lytic mechanism that doesn't result in cell death, thus avoiding the release of cellular danger signals that normally alert the host immune system. Additionally, viruses within the EV lipid bilayer are shielded from neutralizing antibodies and capture by phagocytic cells, reducing the efficacy of the immune response when it does develop. Lastly, the composition and cargo of the EVs sheltering viruses may contribute to immune evasion. EVs universally contain phosphatidylserine lipids, which have been shown to be immunosuppressive and may help reduce immune responses in cells infected by en bloc transmission. Within the EV lumen, cellular immunomodulatory microRNAs (miRNAs) and protein cargos could potentially be codelivered to the target cell. Together, these advantages of en bloc viral transmission may convey significant immune evasion-related advantages (2).

The authors highlight a number of viruses where en bloc transmission has been observed and an interesting observation is that for each disease and corresponding virus, the mechanism by which virions are packaged into $\mathrm{EVs}$ differs. For instance, poliovirus, coxsackievirus B (CVB), encephalomyocarditis virus (ECMV), and enterovirus A71 (EV-A71) are packaged in autophagosomes (11-14). While autophagosomes normally fuse with lysosomes for content degradation, viruses have evolved proteins to interfere with the SNARE complex that mediates this autophagosomelysosome fusion, causing release of virus-containing EVs via autophagosome-plasma membrane fusion. Infectious EV-A71 genomic RNA was separately found in exosomesa small subtype of $\mathrm{EV}$ formed by invagination into the multivesicular body (MVB)—suggesting that some viruses use multiple EV pathways to spread (15). Norovirus, which can cause gastroenteritis, is also spread en bloc within exosomes derived from the MVB (16). HAV and hepatitis E virus (HEV) were both once thought to exist solely as nonenveloped virions, until it was discovered they could interact with ESCRT machinery and be released within exosomes $(17,18)$. HCV is normally released as an enveloped virus, but exosomes containing HCV genomic RNA and proteins without virions have been detected (19). West Nile virus $(\mathrm{WNV})$, Zika virus (ZIKV), and porcine reproductive and respiratory syndrome virus (PRRSV) genome RNA have all been detected with EVs (20-22). Still other viruses use different $\mathrm{EV}$ populations. One agent of viral gastroenteritis, rotavirus, is shed in large plasma-membrane derived EVs (16). Some viruses clearly spread en bloc via an EV pathway but require more characterization to determine the precise EV subtype. These includes Dengue virus (DENV) and Bluetongue virus (BTV), both borne by arthropod vectors $(23,24)$. The ability of viruses to spread via EVs has important public health implications. For instance, methods to detect viruses in blood may be unable to identify viruses enclosed within EVs, and those EV-packaged viruses may be less sensitive to traditional viral neutralization protocols.

Finally, is as so common in science, the mechanism of en bloc transmission raises nearly as many questions as it answers. Paramount among these is the question of how EVs deliver cargo proteins and nucleic acids to target cells. In the context of en bloc transmission, the viruses within the EVs need to release their nucleic acids into the cytosol of the target cell in order to replicate; as a result, fusion between the virus-containing EV and a cellular membrane is a prerequisite for productive viral infection. The authors propose two mechanisms through which this can occur-receptor-dependent fusion and receptorindependent fusion. In receptor-dependent fusion, a viral fusion protein (often called a viral 'envelope' or 'spike' protein) from a circulating virus or a human endogenous 
retrovirus could become embedded in the $\mathrm{EV}$ membrane and impart fusogenic potential to that EV. Indeed, this mechanism for imparting fusion potential to EVs has been demonstrated with multiple viral fusion proteins including those of vesicular stomatitis virus and influenza. In receptor-independent fusion, the authors propose a nonspecific, direct fusion of the EV membranes with a cellular membrane. Despite receptor-independent fusion of EVs being widely hypothesized, the mechanistic details of this route of fusion remain very poorly defined. Understanding how EVs fuse with target cells is key to en bloc transmission and receptor-independent fusion-if it occurs has the potential to expand upon the natural tropism of viruses by bypassing the receptors used to infect cells as free virions. Another fascinating question raised by this review is that of the evolutionary origins of viruses and EVs. The extensive overlap in size, composition, and biogenesis of EVs and enveloped viruses has led to the 'progressive hypothesis' or 'escape hypothesis' of viral evolution that proposes that ancestral viruses were nucleic acids that evolved the ability to move around the genome and then exit the cell and selfreplicate (25). Is it possible that ancestral nucleic acid viruses shuttled between cells within ancient EVs? These infectious EVs may have been the first 'primordial' viruses, consisting primarily of self-replicating DNA or RNA packaged into an EV. Based on this hypothesis, the ability of viruses to spread as free virions evolved over time as viruses developed capsid proteins to selectively package and enclose the viral genome and fusion (envelope, spike) proteins to facilitate membrane fusion with target cells. The en bloc transmission reviewed by Kerviel and colleagues thereby could represent a sort of window into the evolutionary past of viruses.

In conclusion, this review presents a convincing case that EVs should be treated seriously as potential infectious agents within the context of viral disease and public health. The potential of viral spread while cloaked in EVs is a significant concern, although the relative safety of blood products in most developed countries suggests that protocols for maintaining safety are adequate for current viruses of concern. However, knowledge of en bloc transmission is essential to developing strategies to inactivate viruses embedded in EVs. Our current understanding of how viruses hijack EVs for their transmission is clearly still incomplete, and we predict that further fascinating examples of overlap between the $\mathrm{EV}$ machinery and viral replication will emerge as research in this area progresses.

\section{Acknowledgments}

Funding: The manuscript was supported by R01 AI140847 (to Dr. Tilton).

\section{Footnote}

Provenance and Peer Review: This article was commissioned by the editorial office, $E x R N A$. The article has undergone external peer review.

Conflicts of Interest: Both authors have completed the ICMJE uniform disclosure form (available at https://dx.doi. org/10.21037/exrna-21-24). The manuscript was supported by R01 AI140847 (to Dr. Tilton). ZT received a young investigator award and scholarship to attend the ISEV 2019 annual meeting and give an oral presentation, but it doesn't have a conflict of interest to this manuscript. The authors have no other conflicts of interest to declare.

Ethical Statement: The authors are accountable for all aspects of the work in ensuring that questions related to the accuracy or integrity of any part of the work are appropriately investigated and resolved.

Open Access Statement: This is an Open Access article distributed in accordance with the Creative Commons Attribution-NonCommercial-NoDerivs 4.0 International License (CC BY-NC-ND 4.0), which permits the noncommercial replication and distribution of the article with the strict proviso that no changes or edits are made and the original work is properly cited (including links to both the formal publication through the relevant DOI and the license). See: https://creativecommons.org/licenses/by-nc-nd/4.0/.

\section{References}

1. Nolte-'t Hoen E, Cremer T, Gallo RC, et al. Extracellular vesicles and viruses: Are they close relatives? Proc Natl Acad Sci U S A 2016;113:9155-61.

2. Urbanelli L, Buratta S, Tancini B, et al. The Role of Extracellular Vesicles in Viral Infection and Transmission. Vaccines (Basel) 2019;7:102.

3. Martins ST, Alves LR. Extracellular Vesicles in Viral Infections: Two Sides of the Same Coin? Front Cell Infect Microbiol 2020;10:593170.

4. Arenaccio C, Chiozzini C, Columba-Cabezas S, 
et al. Exosomes from human immunodeficiency virus type 1 (HIV-1)-infected cells license quiescent CD4+ T lymphocytes to replicate HIV-1 through a Nef- and ADAM17-dependent mechanism. J Virol 2014;88:11529-39.

5. Lenassi M, Cagney G, Liao M, et al. HIV Nef is secreted in exosomes and triggers apoptosis in bystander CD4+ $\mathrm{T}$ cells. Traffic 2010;11:110-22.

6. Khatua AK, Taylor HE, Hildreth JE, et al. Exosomes packaging APOBEC3G confer human immunodeficiency virus resistance to recipient cells. J Virol 2009;83:512-21.

7. Dreux M, Garaigorta U, Boyd B, et al. Short-range exosomal transfer of viral RNA from infected cells to plasmacytoid dendritic cells triggers innate immunity. Cell Host Microbe 2012;12:558-70.

8. Kerviel A, Zhang M, Altan-Bonnet N. A New Infectious Unit: Extracellular Vesicles Carrying Virus Populations. Annu Rev Cell Dev Biol 2021;37:171-97.

9. Altan-Bonnet N, Chen YH. Intercellular Transmission of Viral Populations with Vesicles. J Virol 2015;89:12242-4.

10. Bou JV, Geller R, Sanjuán R. Membrane-Associated Enteroviruses Undergo Intercellular Transmission as Pools of Sibling Viral Genomes. Cell Rep 2019;29:714-723.e4.

11. Chen YH, Du W, Hagemeijer MC, et al. Phosphatidylserine vesicles enable efficient en bloc transmission of enteroviruses. Cell 2015;160:619-30.

12. Robinson SM, Tsueng G, Sin J, et al. Coxsackievirus $\mathrm{B}$ exits the host cell in shed microvesicles displaying autophagosomal markers. PLoS Pathog 2014;10:e1004045.

13. van der Grein SG, Defourny KAY, Rabouw HH, et al. Picornavirus infection induces temporal release of multiple extracellular vesicle subsets that differ in molecular composition and infectious potential. PLoS Pathog 2019; 15:e1007594.

14. Too IH, Yeo H, Sessions OM, et al. Enterovirus 71 infection of motor neuron-like NSC-34 cells undergoes a non-lytic exit pathway. Sci Rep 2016;6:36983.

15. Gu J, Wu J, Fang D, et al. Exosomes cloak the virion

doi: 10.21037/exrna-21-24

Cite this article as: Troyer Z, Tilton JC. Extracellular vesicles as carriers of viruses. ExRNA 2021;3:13. to transmit Enterovirus 71 non-lytically. Virulence 2020;11:32-8.

16. Santiana M, Ghosh S, Ho BA, et al. Vesicle-Cloaked Virus Clusters Are Optimal Units for Inter-organismal Viral Transmission. Cell Host Microbe 2018;24:208-220.e8.

17. Feng Z, Hensley L, McKnight KL, et al. A pathogenic picornavirus acquires an envelope by hijacking cellular membranes. Nature 2013;496:367-71.

18. Nagashima S, Jirintai S, Takahashi M, et al. Hepatitis E virus egress depends on the exosomal pathway, with secretory exosomes derived from multivesicular bodies. J Gen Virol 2014;95:2166-75.

19. Bukong TN, Momen-Heravi F, Kodys K, et al. Exosomes from hepatitis $\mathrm{C}$ infected patients transmit $\mathrm{HCV}$ infection and contain replication competent viral RNA in complex with Ago2-miR122-HSP90. PLoS Pathog 2014;10:e1004424.

20. Zhou W, Woodson M, Neupane B, et al. Exosomes serve as novel modes of tick-borne flavivirus transmission from arthropod to human cells and facilitates dissemination of viral RNA and proteins to the vertebrate neuronal cells. PLoS Pathog 2018;14:e1006764.

21. Zhou W, Woodson M, Sherman MB, et al. Exosomes mediate Zika virus transmission through SMPD3 neutral Sphingomyelinase in cortical neurons. Emerg Microbes Infect 2019;8:307-26.

22. Wang T, Fang L, Zhao F, et al. Exosomes Mediate Intercellular Transmission of Porcine Reproductive and Respiratory Syndrome Virus. J Virol 2018;92:e01734-17.

23. Reyes-Ruiz JM, Osuna-Ramos JF, De Jesús-González LA, et al. Isolation and characterization of exosomes released from mosquito cells infected with dengue virus. Virus Res 2019;266:1-14.

24. Labadie T, Sullivan E, Roy P. Multiple Routes of Bluetongue Virus Egress. Microorganisms 2020;8:965.

25. Forterre P. The origin of viruses and their possible roles in major evolutionary transitions. Virus Res 2006;117:5-16. 